\title{
Monitoring and Risk Assessment of linear Infrastructures
}

\author{
$\underline{\text { Rene Griesbach }^{1}}$, Oscar Friedel ${ }^{2}$ \\ ${ }^{1}$ Planet Labs Germany GmbH, 10719 Berlin, Germany \\ ${ }^{2}$ Kayrros, 33 Rue La Fayette, FR 75009, Paris, France
}

doi: https://doi.org/10.21467/abstracts.93.105

\begin{abstract}
Despite improving technologies, the intensifying use of natural resources increases the relevant risks for the environment, public health and business operations. Technological developments in the field of risk assessment become, therefore, essential to our economy and overall well-being. Planet and Kayrros are two relatively new players in the geospatial market, specializing in Remote Sensing technologies which are increasingly used to monitor risks threatening the linear infrastructure networks of the Oil and Gas sector. Planet builds, owns and operates the largest ever fleet of Earth observation satellites. More than 140 Planet satellites orbiting around the Earth. These are able to provide an almost full image in high spatial resolution of the entire land surface of the Earth every day. Identified hot spots, which need even higher temporal and spatial resolution, can be monitored up to ten times per day with a spatial resolution of up to $50 \mathrm{~cm}$. The resulting satellite data are available only hours after sensing and easily accessible via web-portal or API.

Kayrros uses Planet imagery to enable power line and pipeline transmission operators to anticipate risks and save resources through its latest automatic monitoring solution for extensive linear infrastructure. Based on the automatic processing of daily satellite data, Kayrros' proprietary AI technology helps operators reducing the frequency of on-site inspections while increasing the safety levels of the surrounding communities. By delivering near real-time measurements of risk-escalating parameters in any location in the world, ranging from soil temperature to vegetation coverage.

The global approach of Planet and Kayrros, based on diverse data sources including daily acquisitions from the Planet-Scope and SkySat constellations, enables the coverage of extensive risk sources such as:

$>$ Wildfires caused by the vegetation encroachment surrounding power lines

$>$ Power line damage caused by temporary structures

$>$ Pipeline leakage caused by accidental damage during groundworks

$>$ at any point on Earth.
\end{abstract}

In the speech, the basic technical specs of the repeated data collection by satellites, the way how they are provided and used in conjunction with other data, and the type of risk related information provided will be addressed, based on real use cases.

For example, by analysing the same scene in two distinct satellite images, captured consecutively from different angles, Kayrros generates digital twins of the surrounding vegetation, measures its growth and emitts alerts in case of potential contact with the high-voltage line network. Alternatively, by processing satellite radar images and applying proprietary computer vision algorithms, Kayrros detects risky groundworks near the sensitive assets and eliminates permanent structures, noise and vehicles from the relevant SAR satellite imagery. Such advanced technologies enable the adoption of data-driven risk management processes and replace assumptions with real measurements. Thus, they deliver substantialbenefits to day-to-day risk assessments, including:

$>$ Increased safety for the communities and the environment surrounding linear infrastructure through active monitoring of risks leading to fire and pollution.

Reduced inspection costs of vast linear infrastructure and minimized liability risks.

(C) 2020 Copyright held by the author(s). Published by AIJR Publisher in "Abstracts of The Second Eurasian RISK-2020 Conference and Symposium" April 12- 19, 2020, Tbilisi, Georgia. Jointly organized by AMIR Technical Services LLC, Georgian Technica University, Institute of Geography (Kazakhstan) and Russian Institute of Petroleum Geology and Geophysics.

Aij. DOI: 10.21467 /abstracts.93 
The Second Eurasian RISK-2020 Conference and Symposium

Reinforcement of the maintenance and the operator's technical support system through automated checks for sensitive structures.

$>$ Enhanced communication throughout the operator's organization through tailor-made data visualization dashboards bringing engineering, HSE and management teams together.

$>$ Reduced outages and disruptions for criticalassets of the Oil \& Gas industry through early intervention alerts. 